\title{
RESEARCH PAPER \\ FACTORIAL EXPERIMENTS FOR PRODUCT QUALITY IMPROVEMENT IN A GHANAIAN MANUFACTURING FIRM
}

\author{
F. L. Larkai ${ }^{1}$ and S. M. Sackey ${ }^{2}$ \\ ${ }^{1}$ Marine Engineering Department, Regional Maritime University, Ghana \\ ${ }^{2}$ Department of Mechanical Engineering, KNUST, Kumasi, Ghana.
}

\begin{abstract}
It is important that the quality of manufactured products meets the expectations of those who use them. There are many tools available to the quality engineer seeking to achieve this goal. This paper discusses improvements in product quality in a Ghanaian edible oil processing firm achievable through factorial experiments. The focus is on input material quality and its constituents, namely \% ripe, \% overripe, and \% rotten of the raw palm fruit. The proportions of the constituents necessary to achieve acceptable levels of product quality, expressed in terms of acceptable levels of the Free Fatty Acid (FFA) content and the Moisture Content (MC), are investigated through factorial experiments. Results show that a high percentage of the overripe palm fruits has a consistently negative effect on overall product quality (i.e. the output FFA and MC levels). The MC offers more flexibility than the FFA in terms of the number of factor combinations that give ideal results. This makes FFA more critical to quality and the parameter to watch from the probability point of view. However, the best results are achieved when overripe is held low in combination with relatively low values of the other two factors, followed by low overripe in combination with relatively low values of the other two factors.
\end{abstract}

Keywords: Product, quality, engineering, factorial experiments

\section{INTRODUCTION}

Crude vegetable oils have been produced in the agro-processing industry from various oil seeds and nuts for a long time. To meet the ever growing demand for high quality, low-priced, edible oil in an increasingly competitive environment, there is the need to use scientific methods to optimise the quality of the product.
Adu-Amankwa (1999) researched into quality control in the food industry and underlined the importance of addressing three key aspects of quality control namely, raw materials quality, process control and finished product inspection. He particularly emphasized the desirability of getting things right regarding material and process quality so as to make, if possible, fin- 


\section{$51 \quad$ Sackey and Larkai}

ished product inspection unnecessary. Sackey and Powell (2006a) examined the issue of general quality awareness in the Ghanaian engineering manufacturing sector. They discovered that overall, most small and medium scale Ghanaian engineering manufacturing firms are inadequately prepared to compete on quality and there is a widespread lack of awareness of modern quality improvement initiatives. Sackey and Powell (2006b) further investigated designer and manufacturer practices affecting product integrity in the small and medium scale agroprocessing equipment manufacturing sector in Ghana, including materials issues relating to corrosion avoidance. Adomako (1998), on his part, highlighted the need for Ghanaian companies to strive to achieve ISO 9000 certification. Recognizing the central role of product quality and how it is impacted by the design function, he rightly observed that without quality products, a manufacturing firm may not be able to compete in today's marketplace. He, however, failed to recommend how this quality could be realized to benefit a manufacturing company. Moreover, very few studies, if any, are found in the literature that employ designed experiments to analyse product quality issues in the food processing industries.

This research employs factorial experiments as an engineering tool to analyze and improve the quality of processed oil produced by a company in the agro-processing industry, where today the need for uncompromisingly high quality has become one of the driving forces. In this industry, during the processing of raw materials into the final product, certain operating parameters are observed to have a direct or indirect effect on the quality of the product, thus bearing on the final value of the product. This is because, as noted by a study undertaken by the Intermediate Technology Development Group (ITDG) in collaboration with the United Nations Fund for Women (UNIFEM), contamination by agents such as excess FFA and other impurities could be very detrimental to human health (Intermediate Technology Development Group, 1993). Optimising this quality is of great im- portance to manufacturing industries because of its crucial link to the health of the consumers of their products.

In referring to optimum product quality as in the preceding paragraph, the relevant Ghana National Standards relating to the product quality characteristics of interest to this study are:

a) Free Fatty Acid (as palmitic acid) $=5 \%$ maximum; and

b) Moisture Content, at $105^{\circ} \mathrm{C}=0.25 \%$ maximum [Ghana Standard, GS 223]

In addition, information from expert food scientists indicate that whereas excess moisture content tends to limit the shelf life of the oils, excess free fatty acid is more potentially detrimental to human health but it is not as obvious as the latter.

\section{Factorial experimental design}

Factorial design of experiments as a scientific method used in investigating product quality is a relatively new concept in developing countries. In the more advanced countries it has been applied in the manufacturing and service industries to improve the quality of products and processes (Antony, 1998). The procedure offers the engineer a valid basis for developing an empirical model of systems under investigation, and is now considered one of the most effective practical tools used in running experiments. Its strategy focuses on planning, conducting, analysing and interpreting experiments so that valid conclusions can be established efficiently and economically.

In this study input parameters affecting the quality of edible oil product from an agroprocessing plant are monitored using the factorial experimental design procedure. The expected outcome is a determination of optimum levels of the input parameters necessary to achieve good product quality.

Objectives of the study

The objectives of the study are to investigate 
the effects and interactions of various factors on FFA and MC, to determine the levels and combinations of the variables that yield optimum product quality, and to derive a quantitative model relating product quality to the input parameters.

\section{METHODOLOGY}

\section{Experimental methods}

A full factorial design with three factors namely, percent rotten $(R)$, percent, overripe $(O)$, and percent ripe $(P)$ in the total fruit mix of the charge for oil production, each with two levels, was employed as the essential experimental design scheme in this work. Table 1 displays the design factors and their levels used in the experiment. From these, several parameters were determined.

Two product quality characteristics of interest, the free fatty acid and moisture content, were determined from samples of the oil using a $2^{k}$ design with $k=3$, thus giving 8 factor-level combinations (runs). In doing this, and to reduce the influence of the researchers, two observations (i.e. replicates) were taken from an initial randomized pool of a total of 16 measurements at the 8 design points. The two observations taken at each design point were then averaged.

Formula for calculating percent free fatty acid content (\%FFA)

The acidity (free fatty acid) is calculated from the formula (Institiut Penyelidikan Minyak Kelapa Sawit Malaysia, 1986):

$\% F F A=\left(\frac{M W \times V \times C}{m}\right) \times 100$ where:

$m=$ mass of the oil sample taken $(\mathrm{g})$

$M W=$ molecular weight of acid (g/k-mole)

$V=$ volume of alkali solution used $(\mathrm{ml})$

$C=$ Concentration of the alkali solution (moles $/ \mathrm{mls}$ )

Formula for calculating percent moisture content (\%MC)

This procedure determines the moisture and any other volatile materials under the conditions of the test. The method involves heating the oil sample in an oven at $103 \pm 2{ }^{\circ} \mathrm{C}$. The moisture content, $M C$, is calculated from the formula (Institiut Penyelidikan Minyak Kelapa Sawit Malaysia, 1986):

$$
\% M C=\left(\frac{m_{d o}-m_{d d}}{m_{d o}-m_{d}}\right) \times 100
$$

where:

$m_{\mathrm{d}}=$ mass of dish

$m_{\mathrm{do}}=$ mass of dish and oil before drying

$m_{\mathrm{dd}}=$ mass of dish and oil after drying.

\section{Calculation of the effects}

The formula for calculating the main effect of Percent Rotten $\left(E_{R}\right)$ is given as:

$$
\begin{aligned}
& E_{R=1 / 4}\left(\left(\mathrm{Q}_{2}+\mathrm{Q}_{4}+\mathrm{Q}_{6}+\mathrm{Q}_{8}\right)-\left(\mathrm{Q}_{1}+\mathrm{Q}_{3}+\mathrm{Q}_{5}+\right.\right. \\
& \left.\left.\mathrm{Q}_{7}\right)\right)
\end{aligned}
$$

where $Q_{\mathrm{i}}$ refers to the $\%$ FFA response at the $i^{\text {th }}$ run point, obtained from two replicates $R E P 1$ and $R E P 2$ (Table 2). Similarly, the main effect of $\%$ Overripe $\left(E_{O}\right)$ is given as:

Table 1: Design factors

\begin{tabular}{llll}
\hline Level & Rotten $(\mathbf{R})(\boldsymbol{\%})$ & Overripe $(\mathbf{O})(\boldsymbol{\%})$ & Ripe $(\mathbf{P})(\boldsymbol{\%})$ \\
\hline Low $(-)$ & 1.215 & 5.512 & 82.338 \\
High $(+)$ & 2.667 & 7.136 & 85.427 \\
\hline
\end{tabular}




\section{Sackey and Larkai}

$E_{o=}{ }^{1 / 4}\left(\left(\mathrm{Q}_{3}+\mathrm{Q}_{4}+\mathrm{Q}_{7}+\mathrm{Q}_{8}\right)-\left(\mathrm{Q}_{1}+\mathrm{Q}_{2}+\mathrm{Q}_{5}+\right.\right.$

Q6)

And that of $\%$ Ripe $\left(E_{P}\right)$ is:

$E_{p=1 / 4}\left(\left(\mathrm{Q}_{5}+\mathrm{Q}_{6}+\mathrm{Q}_{7}+\mathrm{Q}_{8}\right)-\left(\mathrm{Q}_{1}+\mathrm{Q}_{2}+\mathrm{Q}_{3}+\right.\right.$

$\left.\mathrm{Q}_{4}\right)$ )

The $R \times O, R \times P, O \times P$ and $R \times O \times P$ interactions respectively, follow (Box et al, 1978):

$I_{R O}=R \times O=1 / 4\left(\left(\mathrm{Q}_{1}+\mathrm{Q}_{4}+\mathrm{Q}_{5}+\mathrm{Q}_{8}\right)-\left(\mathrm{Q}_{2}+\mathrm{Q}_{3}+\right.\right.$ $\left.\mathrm{Q}_{6}+\mathrm{Q}_{7}\right)$ )

$I_{R P}=R \times P=1 / 4\left(\left(\mathrm{Q}_{1}+\mathrm{Q}_{3}+\mathrm{Q}_{6}+\mathrm{Q}_{8}\right)-\left(\mathrm{Q}_{2}+\mathrm{Q}_{4}\right.\right.$

$\left.\left.+\mathrm{Q}_{5}+\mathrm{Q}_{7}\right)\right)$

$I_{O P=} O \times P=1 / 4\left(\left(\mathrm{Q}_{1}+\mathrm{Q}_{2}+\mathrm{Q}_{7}+\mathrm{Q}_{8}\right)-\left(\mathrm{Q}_{3}+\mathrm{Q}_{4}\right.\right.$

$\left.+\mathrm{Q}_{5}+\mathrm{Q}_{6}\right)$ )

$I_{R O P=} R \times 1 / 4\left(\left(\mathrm{Q}_{2}+\mathrm{Q}_{3}+\mathrm{Q}_{5}+\mathrm{Q}_{8}\right)-\left(\mathrm{Q}_{1}+\mathrm{Q}_{4}\right.\right.$

$\left.\left.+\mathrm{Q}_{6}+\mathrm{Q}_{7}\right)\right)$

The general regression model (Montgomery, 2001) used in calculating the predicted values is

$y=\beta_{0}+\beta_{1} \chi_{1}+\beta_{2} \chi_{2}+\beta_{3} \chi_{3}+\beta_{12} \chi_{1} \chi_{1}+\beta_{13} \chi_{1} \chi_{3}+$

$\beta_{23} \chi_{2} \chi_{3}+\beta_{123} \chi_{1} \chi_{2} \chi_{3}$

where the factors $R, O$, and $P$ are represented by coded variables $x_{1}, x_{2}$, and $x_{3}$, and the $R O$, $R P$, and $O P$ interactions are $x_{1} x_{2}, x_{1} x_{3}$, and $x_{2} x_{3}$ respectively. The $R O P$ interaction is $x_{1} x_{2} x_{3}$. The low and high levels of each factor are assigned the values $x_{\mathrm{j}}=-1$ and $x_{\mathrm{j}}=+1$, respectively.

\section{Calculation of the standard errors}

In calculating the standard errors, the following formulae, from Box et al (1978), were employed:

Pooled Variance

$$
s^{2}=\Sigma d_{i}^{2} / 2 g \quad(g=8)
$$

Variance of an effect
$V($ effect $)=\frac{4}{N} \sigma^{2}$

$(N=16)$

Standard Error of an effect $(\mathrm{Se})=\sqrt{V(\text { Effect })}$

where, $\sigma$, the standard deviation, is estimated by $S$, and $g=$ number of runs, and $N=$ total number of observations at the 8 design points. $d_{\mathrm{i}}$ is the deviation at the $i$ th run point.

\section{Experimental results}

The raw experimental results for both FFA and MC responses are displayed in Tables 2 and 3 below, with plus and minus signs for the $2^{3}$ design. REP means replicate, and RUNPT means run point. The average (AV.) and the deviation $\left(d_{\mathrm{i}}\right)$ at each run point are included.

\section{RESULTS AND DISCUSSION}

Significant effects and interactions for free fatty acid (FFA) response

Table 4 shows a summary of the results for the FFA response, including the factor effect estimates and an estimate of the standard error.

Comparison of each nominal factor effect estimates with the standard error indicates that the interaction effects $I_{R O P}, I_{R P}, I_{O R}$ and the main effect $E_{O}$ are significant. The existence of these interactions means that the main effects $E_{R}, E_{P}$ and $E_{O}$, on their own, do not carry much meaning.

Overripe, $O$, appearing in three significant interactions and tending to increase $F F A$ levels whenever it is present, means that it is an important factor needing close monitoring. It would thus seem prudent to keep Overripe $(O)$ low, in order to reduce overall $F F A$ levels. It is clear that for FFA a low level of Overripe $(O)$, a high level of Rotten $(R)$ and a high level of Ripe $(P)$ (i.e. $\left.R^{+} O^{-} P^{+}\right)$offers the best combination for optimum results.

The significant effects and interactions for moisture content (MC) response

Table 5 displays the results for the factor effect 
Factorial experiments for product quality improvement... 54

Table 2: Factor combinations and experimental results for FFA response

\begin{tabular}{lllllllr}
\hline \multicolumn{7}{c}{ CODED RESPONSE } \\
\hline FUNPT, i & $R$ & $O$ & $P$ & $R E P 1$ & $R E P 2$ & AV. & $d_{i}$ \\
1 & - & - & - & 1.40 & 1.30 & 1.35 & 0.10 \\
2 & + & - & - & 1.54 & 1.58 & 1.56 & -0.04 \\
3 & - & + & - & 1.52 & 1.55 & 1.53 & -0.03 \\
4 & + & + & - & 1.73 & 1.78 & 1.76 & -0.06 \\
5 & - & - & + & 1.47 & 1.83 & 1.65 & -0.36 \\
6 & + & - & + & 1.15 & 1.11 & 1.13 & -0.04 \\
7 & - & + & + & 1.77 & 1.29 & 1.53 & 0.48 \\
8 & + & + & + & 1.80 & 1.95 & 1.88 & -0.15 \\
$F F A_{M}$ & & & & & & 1.55 & \\
\hline
\end{tabular}

Table 3: Factor combinations and experimental results for $\mathrm{MC}$ response

\begin{tabular}{lllllllr}
\hline \multicolumn{7}{c}{ CODED RESPONSE } \\
MC., Q (\%) \\
\hline RUNPT, i & $R$ & $O$ & $P$ & $R E P 1$ & REP2 & AV. & $d_{i}$ \\
1 & - & - & - & 0.02 & 0.03 & 0.025 & -0.01 \\
2 & + & - & - & 0.05 & 0.04 & 0.045 & 0.01 \\
3 & - & + & - & 0.06 & 0.05 & 0.055 & 0.01 \\
4 & + & + & - & 0.02 & 0.04 & 0.030 & -0.02 \\
5 & - & - & + & 0.02 & 0.03 & 0.025 & -0.01 \\
6 & + & - & + & 0.04 & 0.03 & 0.035 & 0.01 \\
7 & - & + & + & 0.03 & 0.02 & 0.025 & 0.01 \\
8 & + & + & + & 0.03 & 0.04 & 0.035 & -0.01 \\
$M C_{M}$ & & & & & & 0.034 & \\
\hline
\end{tabular}

Table 4: Summary of effects for FFA response

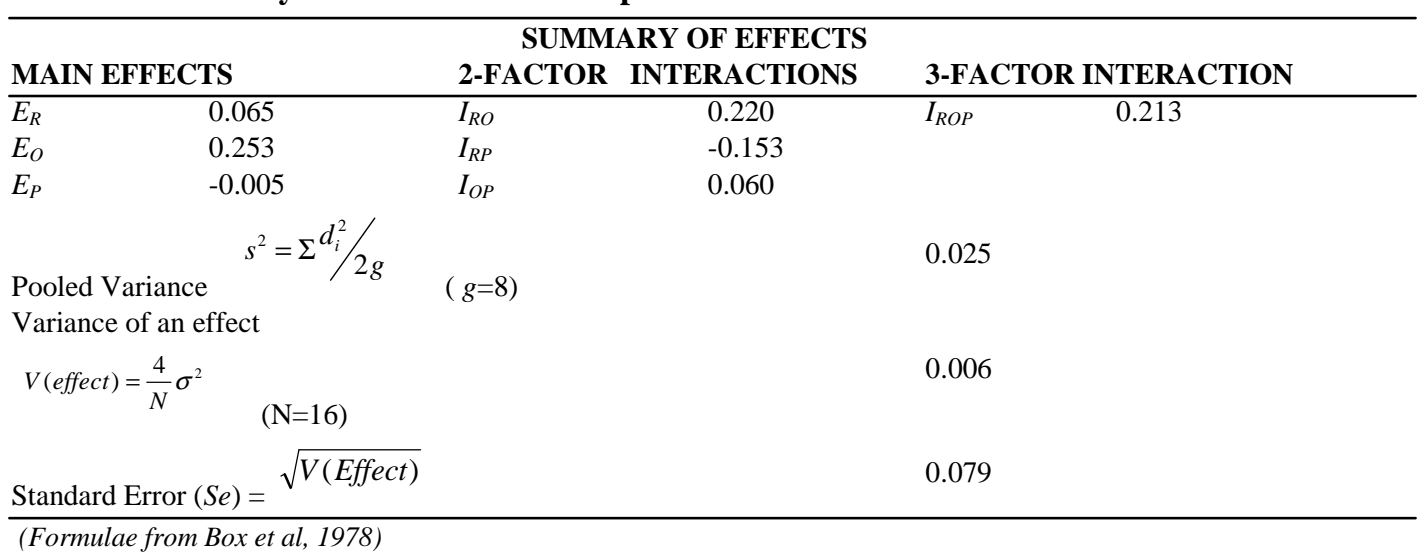


Table 5: Summary of effects for MC response

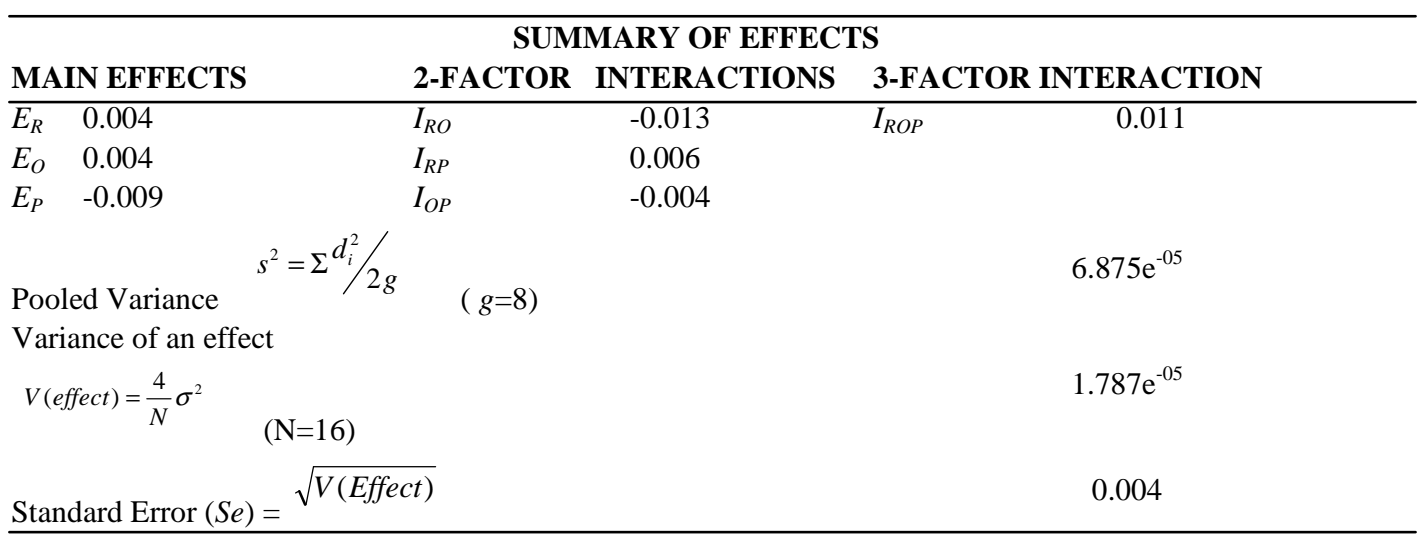

estimates for the moisture content response. As before, it includes Standard Error calculations.

With a standard error of 0.004 , the effects $E_{P}$, $I_{R O}, I_{R P}$, and $I_{\mathrm{ROP}}$ are seen to be significant. The existence of the $R O$ and $R P$ interactions means that each main effect cannot be interpreted separately. The effect of the significant threefactor interaction is to increase moisture content $(M C)$, but the two-factor interaction $R O$ tends to reduce this effect, revealing $P$ as the adverse factor in the $R O P$ interaction.

Further, though $P$ again interacts with $R$ to increase $M C$, its positive effect in $E_{P}$ more than offsets these increases. So, for $M C$, it can be seen that a combination of low $O$, low $R$ and high $P\left(R^{-} O^{-} P^{+}\right)$offers a best scheme to minimize $M C$.

Locating the overall optimum point(s) - Factor interaction plots

Following the above preliminary discussions, interaction plots (Fig. 1) are now used to help locate the overall optimum point.

Fig. 1 confirms that the factor combination that gives the best performance for FFA is $\mathrm{R}^{+} \mathrm{O}^{-} \mathrm{P}^{+}$, and that $O$ must always be low, to ensure low values of FFA. Further, if we are not operating at the optimum level $\left(\mathrm{R}^{+} \mathrm{O}^{-} \mathrm{P}^{+}\right)$then $R$ must be low, otherwise FFA levels would be high.

Fig. 2 shows that for MC there are three possibilities for best performance: $\mathrm{R}^{-} \mathrm{O}^{-} \mathrm{P}^{-}, \mathrm{R}^{-} \mathrm{O}^{-} \mathrm{P}^{+}$, and $\mathrm{R}^{-} \mathrm{O}^{+} \mathrm{P}^{+}$, revealing $\mathrm{R}^{-} \mathrm{O}^{-}$as having twice a probability of occurrence as $\mathrm{R}^{-} \mathrm{O}^{+}$. We would thus prefer to have $\mathrm{R}^{-} \mathrm{O}^{-} \mathrm{P}^{*}$ (the asterisk indicating either a "-" or a "+"). As in the case of $F F A$, it is again clear that for best $M C$ results, the factor $O$ must be low.

In summary (Table 6), the above analyses show that the optimum factor level combination for FFA $\left(R^{+} O^{-} P^{+}\right)$and those for $M C\left(\left(R^{-} O^{-} P^{+}\right),\left(R^{-}\right.\right.$ $\left.O^{-} P^{-}\right)$, and $\left.\left(R^{-} O^{+} P^{+}\right)\right)$, are not coincident. For example, whereas for $M C$ the combination $\left(R^{-}\right.$ $O^{-} P^{+}$) produces best results, for $F F A$ it produces one of the worst outcomes (1.65\% FFA). Remedy could be found in the combination $\left(R^{+} O^{-} P^{+}\right)$, which, in addition to representing the best scheme for $F F A$, produces acceptable results as well for $M C(0.035 \%)$, or in the combination $\left(\mathrm{R}^{-} \mathrm{O}^{-} \mathrm{P}^{-}\right)$which, in addition to representing a best scheme for $M C$, produces acceptable results $(1.35 \%)$ as well for $F F A$. Since, unlike $M C, F F A$ is consistently averse to high overripe $(O)$ levels the combination $\left(R^{-} O^{+} P^{+}\right)$, though a best scheme for $M C$, should not well for $F F A$. Since unlike $M C, F F A$ is consistently averse to 
Factorial experiments for product quality improvement... 56
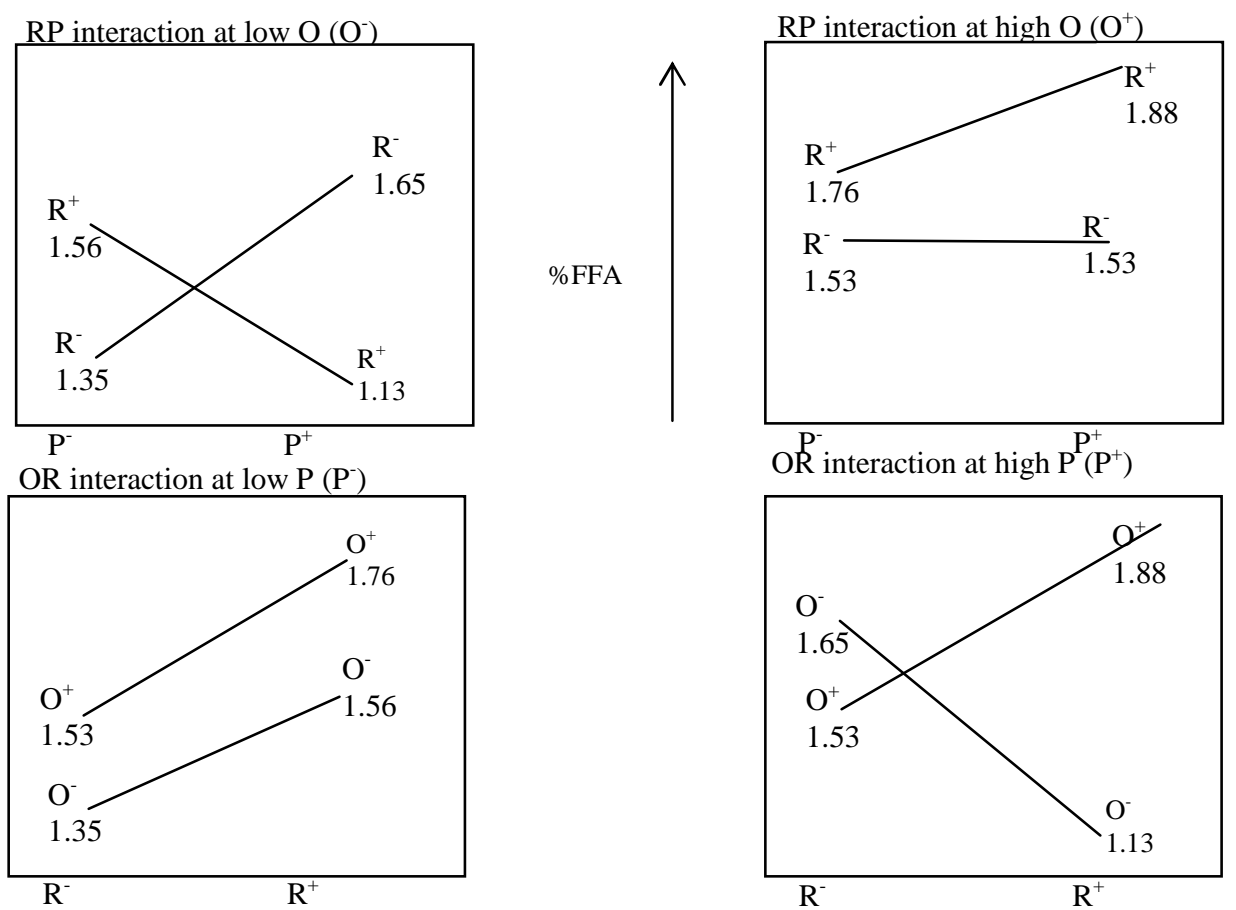

OR interaction at low $\mathrm{P}\left(\mathrm{P}^{-}\right)$

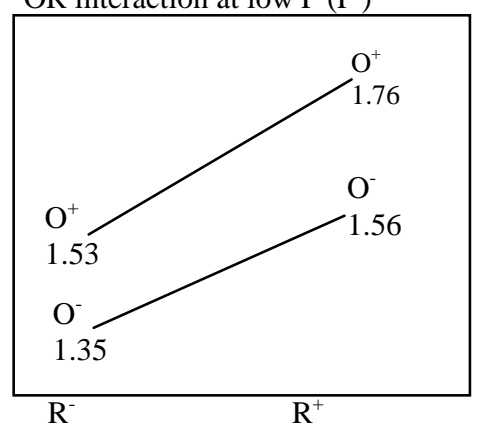

Fig. 1: Free fatty acid factor interaction plots

$\mathrm{RP}$ interaction at low $\mathrm{O}\left(\mathrm{O}^{-}\right)$
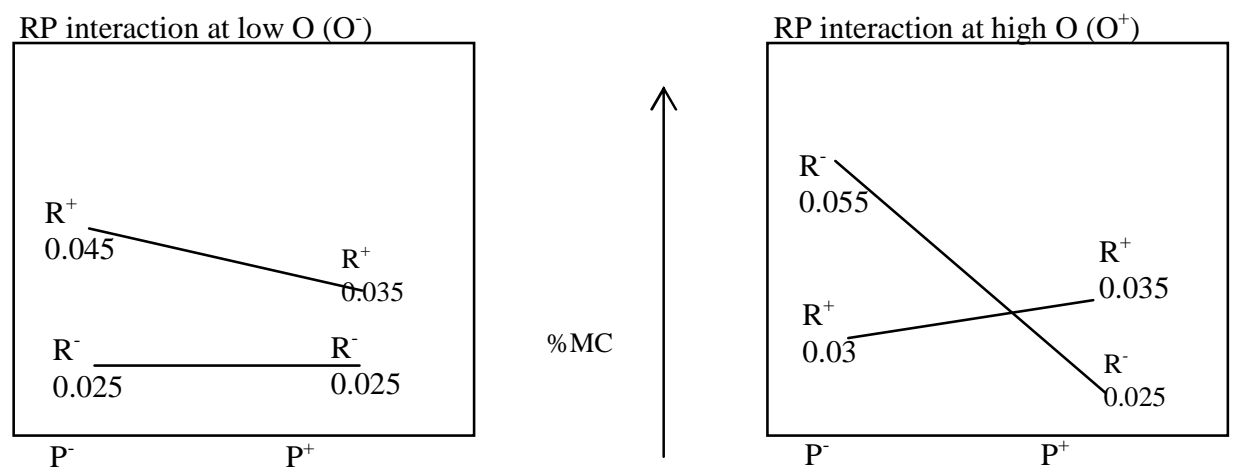

OR interaction at low $\mathrm{P}\left(\mathrm{P}^{-}\right)$

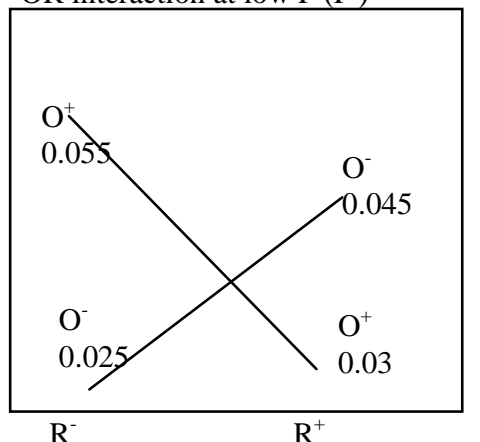

Fig. 2: Moisture content factor interaction plots

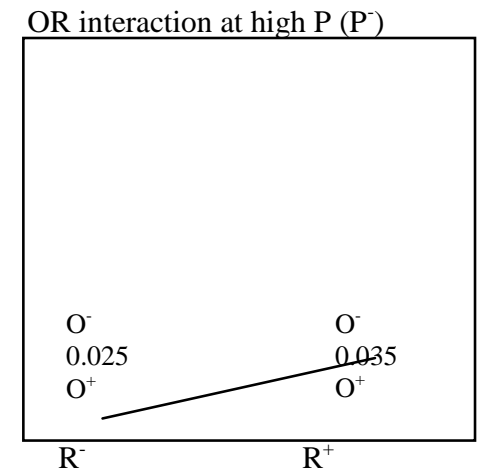

Journal of Science and Technology @ KNUST August 2015 
high overripe $(O)$ levels the combination $\left(R^{-}\right.$ $O^{+} P^{+}$), though a best scheme for $M C$, should not be used at all under any circumstances. Outside the optimum for $F F A$, a low \%Ripe $(P)$ offers higher probability for lower $F F A$ levels than a higher \%Ripe $(P)$. The factor combination for overall best performance would be chosen as $\left(R^{-} O^{-} P^{-}\right)$, followed by $\left(R^{+} O^{-} P^{+}\right)$if $M C$ control is the overriding factor. If $F F A$ control is of greater interest then use $\left(R^{+} O^{-} P^{+}\right)$, followed by $\left(R^{-} O^{-} P^{-}\right)$.

\section{Development and validation of the predicted model}

The significant effects and interactions are used to develop an empirical model for each response. This is done by employing equation 10 . Since the factors Rotten $(R)$, Ripe $(P)$ and the interaction Overripe-Ripe $(O P)$ do not significantly affect the $F F A$ response, the fitted model (using Equation 10) for $F F A\left(F_{M O D}\right)$, neglecting the $E_{R}, E_{P}$ and $I_{O P}$ terms, becomes

$$
\begin{aligned}
F_{\text {(MSOD }}= & 1.547+(0.253 / 2) E_{O}+ \\
& (0.220 / 2) I_{R O}+(0.153 / 2) I_{R P}+ \\
& (0.213 / 2) I_{\text {ROP }} \quad \ldots \ldots \ldots .(11)
\end{aligned}
$$

and that for the $M C$ becomes

$$
\begin{aligned}
M_{(M O D)}= & 0.034+(0.019 / 2) E_{P}+ \\
& (0.011 / 2) I_{R 0}-(0.006 / 2) I_{R P}+ \\
& (0.011 / 2) I_{R 0 P} \quad \ldots \ldots \ldots \ldots(1)
\end{aligned}
$$

Values are generated for both $F F A$ and $M C$ using equations 11 and 12 respectively. The estimated percentage errors there from are displayed in Tables 7 and 8 .

The two tables indicate a good agreement between the calculated values and the experimental data, since the results obtained for percentage error is within the experimental error. Hence, the model obtained for both responses can be used to predict the responses of various ranges of the inputs. 
Factorial experiments for product quality improvement... 58

Table 7: Verification of FFA model

\begin{tabular}{rrrrrrrcc}
\hline $\mathbf{R}$ & $\mathbf{O}$ & $\mathbf{P}$ & \multicolumn{1}{c}{ RO } & \multicolumn{1}{c}{ RP } & ROP & $\mathbf{F}_{\text {EXPT }}(\boldsymbol{\%})$ & $\mathbf{F}_{\text {MOD }}(\%)$ & ERROR (\%) \\
\hline-1 & -1 & -1 & 1 & 1 & -1 & 1.37 & 1.35 & 1.21 \\
1 & -1 & -1 & -1 & -1 & 1 & 1.70 & 1.64 & 3.22 \\
-1 & 1 & -1 & -1 & 1 & 1 & 1.54 & 1.63 & -5.88 \\
1 & 1 & -1 & 1 & -1 & -1 & 1.60 & 1.65 & -3.27 \\
-1 & -1 & 1 & 1 & -1 & 1 & 1.74 & 1.80 & -3.15 \\
1 & -1 & 1 & -1 & 1 & -1 & 1.19 & 1.20 & -1.40 \\
-1 & 1 & 1 & -1 & -1 & -1 & 1.55 & 1.50 & 3.36 \\
1 & 1 & 1 & 1 & 1 & 1 & 1.87 & 1.77 & 4.84 \\
\hline
\end{tabular}

(Figures corrected to two decimal places)

Table 8: Verification of MC model

\begin{tabular}{|c|c|c|c|c|c|c|c|c|c|}
\hline$\overline{\mathbf{R}}$ & $\mathbf{O}$ & $\mathbf{P}$ & $\mathbf{R O}$ & $\mathbf{R P}$ & $\mathbf{O P}$ & ROP & $\mathrm{M}_{\mathrm{EXPT}}(\%)$ & $\overline{M_{M O D}(\%)}$ & $\overline{\text { ERROR (\%) }}$ \\
\hline-1 & -1 & -1 & 1 & 1 & 1 & -1 & 0.02 & 0.02 & 6.25 \\
\hline 1 & -1 & -1 & -1 & -1 & 1 & 1 & 0.03 & 0.03 & 1.92 \\
\hline-1 & 1 & -1 & -1 & 1 & -1 & 1 & 0.06 & 0.05 & 2.27 \\
\hline 1 & 1 & -1 & 1 & -1 & -1 & -1 & 0.03 & 0.03 & 2.08 \\
\hline-1 & -1 & 1 & 1 & -1 & -1 & 1 & 0.03 & 0.03 & -2.08 \\
\hline 1 & -1 & 1 & -1 & 1 & -1 & -1 & 0.04 & 0.04 & -3.57 \\
\hline-1 & 1 & 1 & -1 & -1 & 1 & -1 & 0.03 & 0.03 & -2.08 \\
\hline 1 & 1 & 1 & 1 & 1 & 1 & 1 & 0.04 & 0.04 & -3.57 \\
\hline
\end{tabular}

(Figures corrected to two decimal places)

\section{CONCLUSION}

This paper focused on quality improvement by employing factorial design of experiments to determine the significance of three primary factors (\% Ripe, \% overripe and \% rotten palm fruits) and their interactions on the quality of oil produced in terms of the free fatty acid and moisture content. The objective of determining the levels of the variables that can give optimum product quality and establishing a quantitative model relating product quality to the input variables, have been addressed. Several useful conclusions can be drawn from the study with regard to product quality:

1. A significant 3-Factor interaction exists for both responses (FFA and $M C)$.
2. The best product quality observed in the study is $1.13 \% F F A$, and $0.025 \% M C$ whilst the worst is $1.88 \% F F A$, and $0.055 \%$ MC. By the National Standards presented under the objectives of the study, these are all acceptable figures.

3. Quantitative equation models, generated for the FFA and $M C$ responses in equations 11 and 12 , are found to compare favourably with the experimental data.

Major new findings include:

i. For any combination of the other two factors, a low level of Overippe always produces an an adverse effect, therefore it 


\section{Sackey and Larkai}

i. must never be held at the high level. In other words, an increase in percentage overripe palm fruits has a negative effect on the overall product quality; thus keeping the $\%$ overripe content low improves the quality of the oil produced.

ii $F F A$ is consistently and significantly averse to high Overripe $(O)$ levels, which must always be low to ensure low values of FFA; thus the combination $\left(\mathrm{R}^{-} \mathrm{O}^{+} P^{+}\right)$, though a best scheme for $M C$, should not be used at all under any circumstance.

iii $M C$ offers more flexibility than $F F A$ in terms of the number of factor combinations that give ideal results. This makes FFA more critical to quality and the parameter to watch from the probability point of view.

iv. Outside of the FFA optimum (i.e. $R^{+} O^{-} P^{+}$), a low \%Ripe $(P)$ offers higher probalitity for lower $F F A$ levels than a higher \% Ripe $(P)$. Based on the arguments in section 1.1 regarding the comparative importance of excess MC as compared to excess FFA, the factor combination for overall best performance would be chosen as $\mathrm{R}^{-} \mathrm{O}^{-} \mathrm{P}^{-}$.

\section{CONCLUSION}

In conclusion opportunities exist for quality engineering to make a significant impact on manufacturing in general, and on the oilprocessing industry in particular. Accordingly, future research in a similar vein could extend to other agro-based industries, processing the same or similar products to provide opportunity for comparisons to be made.

\section{ACKNOWLEDGEMENT}

Acknowledgement is to the Juabeng Oil Mills Ltd, Ghana, for their support and cooperation in this research.

\section{REFERENCES}

Adomako, B. (1998). The Role of the Engineer in Industry, The Ghana Engineer. Journal of the Ghana Institution of Engineers, 19 (1): 16.

Adu-Amankwa, P. (1999). Quality and Process Control in the Food Industry, The Ghana Engineer. Journal of the Ghana Institution of Engineers, 20(1): 17-20.

Antony, J. and Capon, N. (1998). Teaching Experimental Design Techniques to Industrial Engineers. International Journal of Engineering Education, 14(5): 335-343.

Box, G.P., Hunter, W. G. and Stuart, H. J. (1978). Statistics for experiments: John Wiley \& Sons, Inc, U.S.A.

Ghana Standard (GS) 223, (2005). $2^{\text {nd }}$ Edition, Animal and Vegetable Fats and Oils: - Specification for Edible Palm Oil, Ghana Standards Authority, Accra, Ghana.

Institiut Penyelidikan Minyak Kelapa Sawit Malaysia, (1986). Palm Oil Process Handbook: Part 3- Laboratory and milling control, Palm Oil Research Institute of Malaysia, Malaysia.

Intermediate Technology Development Group, (1993). Oil Processing, Intermediate Technology Publications Ltd, 9 King Street. London WC2E 8HN, United Kingdom.

Montgomery, D. C. (2001). Design and analysis of experiments: John Wiley \& Sons, Inc, USA.

Sackey, S. M. and Powell, J. W. (2006a). "Quality Issues in Small and Medium Ghanaian Engineering Manufacturing Companies", Journal of The Ghana Institution of Engineers, 4(1): 21-28

Sackey, S. M. and Powell, J. W. (2006b), "Improving Product Integrity in Agroprocessing Equipment Manufacture in Ghana”. Journal of the Ghana Institution of Engineers, 4(2):35-44. 